OPEN ACCESS

Edited by:

Bang Shen,

Huazhong Agricultural University,

China

Reviewed by

Ching-Yuan Chen,

Columbia University Medical Center,

United States

Xiaokai Song,

Nanjing Agricultural University, China

*Correspondence:

Longxian Zhang

zhanglx8999@gmail.com.

Specialty section:

This article was submitted to

Infectious Diseases,

a section of the journal

Frontiers in Microbiology

Received: 19 March 2017 Accepted: 06 September 2017

Published: 20 September 2017

Citation:

Wang R, Zhao G, Gong Y and Zhang $L$ (2017) Advances and Perspectives on

the Epidemiology of Bovine Cryptosporidium in China in the Past

30 Years. Front. Microbiol. 8:1823. doi: 10.3389/fmicb.2017.01823

\section{Advances and Perspectives on the Epidemiology of Bovine Cryptosporidium in China in the Past 30 Years}

\author{
Rongjun Wang', Guanghui Zhao'2, Yunya Gong ${ }^{3}$ and Longxian Zhang ${ }^{1 *}$ \\ ${ }^{1}$ College of Animal Science and Veterinary Medicine, Henan Agricultural University, Zhengzhou, China, ${ }^{2}$ College of Veterinary \\ Medicine, Northwest A\&F University, Yangling, China, ${ }^{3}$ College of Animal Science and Technology, Henan University of \\ Science and Technology, Luoyang, China
}

Major progress has been made in understanding the epidemiology of bovine Cryptosporidium in China in the past 30 years. The overall infection rate in that period was $14.50 \%$ (5265/36316), with different prevalence being observed among dairy cattle, yaks, beef cattle, and buffalo. The infection rate declined as the animals' ages increased and the lowest prevalence occurred in winter. Ten Cryptosporidium species and two genotypes have been found in cattle, with Cryptosporidium parvum, C. andersoni, C. bovis, and C. ryanae being the commonest species. Cryptosporidium bovis rather than C. parvum predominated in preweaned dairy cattle, and C. parvum IIA15G1 and IIdA19G1 were the only subtypes detected in dairy cattle. Two subtype families, Ila and Ild, were found in yaks. Population genetic analysis detected an epidemic population structure in C. andersoni, which suggested that the prevalence of $C$. andersoni in China is not attributable to the introduction of dairy cattle. Moreover, C. parvum Ild subtypes probably dispersed from western Asia to other geographic regions based on population genetic analysis of isolates from China, Sweden, and Egypt. Therefore, we hypothesize that Cryptosporidium was introduced into China in the past, and different populations formed progressively in various hosts in response to diverse factors, including the transmission dynamics, geographic isolation, host specificity, and large-scale farming. More epidemiological studies are required to test this hypothesis and to clarify the prevalence and transmission of Cryptosporidium species in China.

Keywords: Cryptosporidium, cattle, genotype, subtype, population structure, China

\section{INTRODUCTION}

Cryptosporidium spp. are important zoonotic agents infecting a wide spectrum of vertebrate hosts (Xiao et al., 2004; Wang et al., 2011b). There is extensive genetic variation within the genus Cryptosporidium. To date, thirty-one recognized species and more than 60 Cryptosporidium genotypes have been discovered (Xiao et al., 2004; Wang et al., 2010; Ryan et al., 2014). Recent studies into the global causes of severe diarrhea in children suggested that Cryptosporidium is the second most important diarrheal pathogen after Rotavirus (Kotloff et al., 2013; Checkley et al., 2015; Vinayak et al., 2015).

Members of the genus Cryptosporidium complete all developmental stages in a single host. Sporulated oocysts, containing four sporozoites, are released from an infected host upon defecation 
(O'Hara and Chen, 2011). Following ingestion by a suitable host, the motile, infective sporozoites are released through a suture in the oocyst wall and parasitize epithelial cells of the gastrointestinal tract or other tissues (Reduker et al., 1985; O'Hara and Chen, 2011). In these cells, the parasites undergo asexual multiplication, which produces Type-I and Type-II merozoites (Current and Reese, 1986). Type-II merozoites ultimately produce either male or female equivalent sexual reproductive stages, microgametocytes and macrogametocytes, respectively (O'Hara and Chen, 2011). After the macrogamonts are fertilized by the microgametes, oocysts are formed and then sporulated in the infected host. Thick-walled and thin-walled representing two different types of oocysts are produced. The former is commonly excreted from the host, and the latter is primarily involved in autoinfection. Cryptosporidium can be transmitted by the fecal-oral route, via either direct contact or ingestion of contaminated food or water (Xiao et al., 2004; Wang et al., 2011c).

Cattle are the mammals in which Cryptosporidium infection is most commonly found, and preweaned calves are considered the most important reservoir for zoonotic infection (Wang et al., 2011b; Li F. et al., 2016). Cryptosporidium parvum, C. bovis, C. andersoni, and C. ryanae are predominantly responsible for bovine cryptosporidiosis, although several other Cryptosporidium species and genotypes are also discovered in cattle, including C. felis, C. hominis, C. suis, C. scrofarum, C. meleagridis, and C. suis-like genotype (Trout and Santín, 2008; Wang et al., 2011b; Zhang et al., 2013; Huang et al., 2014; Robertson et al., 2014). Studies conducted in numerous industrialized nations have suggested that C. parvum is the species most often found in preweaned calves and that it is a significant cause of diarrhea (Wang et al., 2011b). Cryptosporidium bovis and C. ryanae usually infect weaned calves and yearlings, although $C$. bovis is more commonly seen than C. ryanae, but neither are associated with the occurrence of diarrhea (Santín et al., 2008; Wang et al., 2011b). In contrast, C. andersoni is commonly observed in adult cattle and has been associated with gastritis, reduced milk yield, and poor weight gain (Esteban and Anderson, 1995; Wang et al., 2011b).

Cryptosporidium infections are frequently detected in humans and various domestic and wild animals in China (Wang et al., 2008, 2010, 2011c, 2014a; Feng et al., 2012). Among these, cattle are one of the major targets in which Cryptosporidium is studied. To date, 97 papers involving 24 provinces, autonomous districts, and municipalities of China have been published on Cryptosporidium infections in cattle since the first case was reported in Zhou et al. (1985). The present paper focuses on the advances in the molecular epidemiology of bovine Cryptosporidium that have occurred in China in the past 30 years.

\section{Cryptosporidium INFECTION RATE}

These data were calculated from 97 published papers reporting bovine Cryptosporidium infections in China. The overall infection rate was $14.50 \%(5265 / 36316)$, with a prevalence of $13.98 \%$ (4405/31504), 20.92\% (667/3189), 10.47\% (122/1165), and $15.50 \%(71 / 458)$ in dairy cattle, yaks, beef cattle, and buffalo, respectively $\left(\chi^{2}=128.32 ; P<0.01\right)$. The infection rate for Cryptosporidium species was $45.78 \%$ (141/308) in diarrheal calves (Zhou et al., 1985; Chen et al., 1992; Qin et al., 1994; Cui et al., 2014), which was significantly higher than the average prevalence in cattle (14.50\%). A correlation between the prevalence and the age of the animals was observed. In general, the infection rate declined as the age of the animals increased (Guo et al., 1993; Chen et al., 2011; Wang et al., 2011a,b; Mi et al., 2013; Ma et al., 2014). In dairy cattle in Henan Province, the infection rates of Cryptosporidium species were $21.5 \%(172 / 801)$ in preweaned calves, $11.3 \%(86 / 758)$ in 3-11-month-old calves, $5.7 \%(5 / 262)$ in 12-24-month-old heifers, and $1.0 \%(3 / 295)$ in $>24$-monthold adult cattle (Wang et al., 2011a,b). In contrast, only two studies have determined the prevalence of Cryptosporidium across different seasons, and the prevalence was lowest in winter in both dairy cattle (Wang et al., 2011b) and yaks (Mi et al., 2013).

\section{Cryptosporidium SPECIES DISTRIBUTION}

A total of 1690 Cryptosporidium-positive isolates were genotyped and ten Cryptosporidium species and two genotypes were identified, including C. bovis, C. andersoni, C. ryanae, C. parvum, C. xiaoi, C. ubiquitum, C. meleagridis, C. hominis, C. tyzzeri, C. serpentis, C. suis-like genotype, and a new genotype (Table 1). Cryptosporidium bovis (129/364) rather than C. parvum (119/364) was the predominant Cryptosporidium species in preweaned dairy cattle (Wang et al., 2011b; Zhang et al., 2013; Cui et al., 2014; Huang et al., 2014; Qi et al., 2015). The earliest detection of C. bovis was in 1-week-old calves, indicating that the prepatent period was shorter than the previously recorded 10-12 days (Wang et al., 2011b). Cryptosporidium andersoni was most commonly found in heifers and adult cattle (Wang et al., 2011a). In contrast, another two common species, C. bovis and C. ryanae, occurred at different rates in different epidemiology studies. It is noteworthy that C. tyzzeri (formerly Cryptosporidium mouse genotype I) and C. serpentis probably arose from contamination (Chen and Huang, 2012), and the authors stated in the GenBank submissions that identical sequences (DQ855266 and DQ855267) were found in isolates from pigs with a reverse transcription-PCR analysis of the small subunit ribosomal RNA (Chen and Huang, 2007), and several SSU rRNA gene sequences of C. tyzzeri (EU369382, EF025503, EU369384, EU369381, and EU369383) previously isolated from bovine samples have also been deposited in GenBank. Neither C. tyzzeri nor C. serpentis is a known bovine parasite (Wang et al., 2011a,b).

As in dairy cattle, C. parvum, C. andersoni, C. bovis, and $C$. ryanae were also the four Cryptosporidium species most commonly identified in yaks, although several other Cryptosporidium species and genotypes have been detected (Table 1). However, the distribution of different Cryptosporidium species according to yak age is still unclear. A study conducted in Qinghai suggested that C. bovis was the predominant species in yaks $\leq 2$ years old, whereas $C$. parvum was more common 
in older yaks (Mi et al., 2013). In contrast, a recent study in Tibet showed that C. andersoni was predominant in 1-2-yearold yaks, whereas C. bovis was commonly found in older yaks (Li P. et al., 2016). Therefore, more studies are required to clarify the Cryptosporidium distributions according to age in different groups of animals. Two studies that genotyped Cryptosporidiumpositive isolates from beef cattle and buffalo identified only C. andersoni, C. bovis, and C. ryanae (Ma et al., 2015; Qi et al., 2016).

\section{Cryptosporidium SUBTYPES}

Subtyping tools have been used extensively in studies of the transmission of C. hominis, C. parvum, and several other related Cryptosporidium species, including C. meleagridis and
C. ubiquitum, in both humans and animals (Xiao, 2010; Li et al., 2014; Ryan et al., 2014). One of the most frequently used subtyping tools is a DNA sequence analysis of the $60-\mathrm{kDa}$ glycoprotein (gp60, also known as gp40/15) gene (Ryan et al., 2014).

In dairy cattle, a total of 141 C. parvum isolates have been subtyped by sequencing of the gp60 gene (Table 2). Only the IId subtype family was identified, including IIdA15G1 in Gansu Province and the Ningxia Hui Autonomous Region (Cui et al., 2014; Huang et al., 2014; Zhang et al., 2015) and IIdA19G1 in Henan and Heilongjiang Provinces (Wang et al., 2011b; Zhang et al., 2013). Another study described a cryptosporidiosis outbreak caused by C. parvum subtype IIdA15G1 on a dairy farm in northwestern China (Cui et al., 2014). Three C. meleagridis isolates from preweaned calves were subtyped as IIIeA22G2R1, which was not identical to any known C. meleagridis subtype

TABLE 1 | Cryptosporidium species/genotypes identified in dairy cattle, yaks, beef cattle, and buffalo in China.

\begin{tabular}{|c|c|c|c|c|}
\hline Animal & Isolate no. & \multicolumn{2}{|c|}{ Cryptosporidium species/genotype } & Reference \\
\hline Dairy cattle & 1437 & \multicolumn{2}{|c|}{$\begin{array}{l}\text { C. andersoni (457), C. parvum (315), C. bovis (332), C. ryanae } \\
\text { (86), C. tyzzeri (185), C. serpentis (4), C. hominis (24), } \\
\text { C. meleagridis (5), C. bovis + C. ryanae (9), } \\
\text { C. parvum + C. bovis (6), C. parvum + C. ryanae (4), } \\
\text { C. parvum + C. andersoni (3) }\end{array}$} & $\begin{array}{l}\text { Watanabe et al., 2005; Feng et al., 2007; Zhou et al., 2007; Liu } \\
\text { et al., 2009; Su et al., 2011; Wang et al., 2011a,b; Chen and } \\
\text { Huang, 2012; Zhang et al., 2013, 2015; Cui et al., 2014; Huang } \\
\text { et al., 2014; Ma et al., 2015; Qi et al., 2015, } 2016\end{array}$ \\
\hline Yak & 337 & \multicolumn{2}{|c|}{$\begin{array}{l}\text { C. andersoni (75), C. parvum (28), C. bovis (143), C. ryanae } \\
\text { (78), C. ubiquitum (2), C. xiaoi (1), C. suis-like genotype (2), } \\
\text { C. parvum + C. bovis (2), C. bovis + C. ryanae (4), new } \\
\text { genotype (2) }\end{array}$} & $\begin{array}{l}\text { Mi et al., 2013; Ma et al., 2014; Qin et al., 2014; Qi et al., 2015; } \\
\text { Li P. et al., } 2016\end{array}$ \\
\hline Beef cattle & 108 & \multicolumn{2}{|c|}{$\begin{array}{l}\text { C. andersoni (85), C. bovis (16), C. ryanae (6), } \\
\text { C. bovis + C. ryanae (1) }\end{array}$} & Ma et al., 2015; Qi et al., 2016 \\
\hline Buffalo & 40 & \multicolumn{2}{|c|}{ C. bovis (7), C. ryanae (33) } & Ma et al., 2015 \\
\hline Animal & \multicolumn{2}{|c|}{ Cryptosporidium species } & Subtypes & Reference \\
\hline \multirow[t]{2}{*}{ Dairy cattle } & C. parvum & & ॥dA15G1 (86), IdA19G1 (55) & $\begin{array}{l}\text { Wang et al., 2011b; Zhang et al., 2013, 2015; Cui et al., } \\
\text { 2014; Huang et al., } 2014\end{array}$ \\
\hline & C. meleagridis & & IIleA22G2R1 (3) & Zhang et al., 2013 \\
\hline \multirow[t]{2}{*}{ Yak } & C. parvum & & $\begin{array}{l}\text { IdA15G1 (3), IdA18G1 (1), \|dA19G1 (1), IaA15G2R1 (8), } \\
\text { IlaA16G2R1 (2), IlaA14G1R1 (1), IlaA14G2R1 (1), } \\
\text { IlaA16G3R1 (1) }\end{array}$ & Mi et al., 2013; Qi et al., 2015 \\
\hline & C. ubiquitum & & Xlla subtype 2 (1) & Qi et al., 2015 \\
\hline
\end{tabular}

FIGURE 1 I Population structure inferred by Bayesian clustering using multilocus subtype informations of $C$. andersoni. K-means partitional clustering and the
admixture mode were used in STRUCTURE 2.3.4 and the most appropriate number of K was calculated using an ad hoc statistic-based approach implemented in
Structure Harvester v0.6.94 (http://taylorO.biology.ucla.edu/struct_harvest/).


(Zhang et al., 2013). The subtypes of C. parvum in yaks appear to be more heterogeneous than those in dairy cattle. Thirteen Cryptosporidium-positive isolates from yaks in Qinghai Province were identified as family IIa and five subtypes were detected (Mi et al., 2013). In another study, three IId subtypes (IIdA15G1, IIdA18G1, and IIdA19G1) were detected in five yaks from Qinghai, Gansu, and Tibet, and one C. ubiquitum isolate belonged to zoonotic XIIa subtype 2 (Qi et al., 2015). To date, both C. parvum subtype IIdA19G1 and C. ubiquitum XIIa subtype 2 have also been found in humans in China (Wang et al., 2013; Li et al., 2014).

\section{POPULATION GENETICS}

With the development of Cryptosporidium subtyping tools, it has become possible to assess the genetic and population structures of Cryptosporidium species (Xiao and Ryan, 2008). A total of 149 C. andersoni isolates from beef cattle $(n=38)$ and dairy cattle $(n=111)$ were subtyped with a multilocus sequence typing (MLST) tool based on the MS1, MS2, MS3, and MS16 loci. Fourteen MLST subtypes were identified and A4,A4,A4,A1 was the predominant subtype (Wang et al., 2012; Zhao et al., 2013; Qi et al., 2016). To test the possibility that this linkage disequilibrium (LD) was attributable to the clonal expansion of one or more subtype, masking the underlying equilibrium, an LD analysis was conducted using only the MLST subtypes (by considering each group of isolates with the same MLST subtype as one individual (Wang et al., 2012), 14 MLST subtypes were used in this analysis), with LIAN version 3.7. This analysis suggested that the $C$. andersoni population in cattle in China had an epidemic population structure $\left(I_{\mathrm{A}}{ }_{\mathrm{A}}=0.0010, V_{\mathrm{D}}<L\right)$. An analysis using STRUCTURE 2.3.4 with K-means partitional clustering and the admixture model revealed three ancient lineages among the 149 C. andersoni specimens (Figure 1).

Cryptosporidium parvum is another species that has been targeted for the population genetic analysis in cattle. Cryptosporidium parvum IId isolates $(n=111)$ from several species of animals in China, Sweden, and Egypt (Wang et al., 2014b) were subtyped with an MLST tool based on 12 microsatellite, minisatellite, and single-nucleotide polymorphism loci (Wang et al., 2014b). Host adaptation and significant geographic segregation were both observed in the MLST subtypes. A clonal population structure was detected in the C. parvum IId isolates from China and Sweden. Three ancestral lineages and the same RPGR (retinitis pigmentosa GTPase regulator) sequence were shared by the isolates examined (Wang et al., 2014b). The authors concluded that the C. parvum IId subtypes probably dispersed from western Asia to other geographic regions (Wang et al., 2014b; Zahedi et al., 2016).

\section{REFERENCES}

Checkley, W., White, A. C. Jr., Jaganath, D., Arrowood, M. J., Chalmers, R. M., Chen, X. M., et al. (2015). A review of the global burden, novel diagnostics, therapeutics, and vaccine targets for Cryptosporidium. Lancet Infect. Dis. 15, 85-94. doi: 10.1016/S1473-3099(14)70772-8

\section{CONCLUSION AND PERSPECTIVES}

Epidemiological data suggest that Cryptosporidium infections are commonly found in cattle in China. The infection rate declines as the age of the animals increases, and the lowest infection rate occurs in winter. Similar to the species distributions reported in other countries and areas of the world, C. parvum, C. bovis, C. andersoni and C. ryanae are the four commonest Cryptosporidium species in cattle. Cryptosporidium bovis rather than C. parvum was the dominant Cryptosporidium species in preweaned dairy cattle, which differs from the dominant species in this age group in other countries and areas of the world. Uniquely, the C. parvum subtypes identified in dairy cattle were all zoonotic IIdA15G1 or IIdA19G1 (Wang et al., 2014b).

Population genetic analyses of 149 C. andersoni isolates in three published studies confirmed an epidemic population structure, and as proposed in a previous study, these data suggest that the prevalence of $C$. andersoni in China is not attributable to the introduction of dairy cattle (Wang et al., 2012). According to a population genetic analysis, the C. parvum IId subtypes probably dispersed from western Asia to other geographic regions (Wang et al., 2014b). Therefore, we hypothesize that Cryptosporidium was introduced into China at some time in the past, and then different Cryptosporidium populations developed progressively in various hosts in response to diverse factors, including the transmission dynamics, geographic isolation, host specificity, and large-scale farming. More epidemiological studies are required to confirm this hypothesis and to clarify the transmission and public-health impact of Cryptosporidium species in China.

\section{AUTHOR CONTRIBUTIONS}

LZ had the ideal for the review and revised the manuscript. RW wrote the paper. RW, GZ, and YG reviewed and abstracted data from each selected article.

\section{FUNDING}

This study was supported in part by the Key Program of the National Natural Science Foundation of China (31330079), the National Natural Science Foundation of China (31672548, 31302079, and 31572509), the Program for Science and Technology Innovation Talents in Universities of Henan Province (16HASTIT018), and the Natural Science Foundation of Henan Province (162300410129).

Chen, F., and Huang, K. (2007). Prevalence and phylogenetic analysis of Cryptosporidium in pigs in eastern China. Zoonoses Public Health 54, 393-400. doi: 10.1111/j.1863-2378.2007.01078.x

Chen, F., and Huang, K. (2012). Prevalence and molecular characterization of Cryptosporidium spp. in dairy cattle from farms in China. J. Vet. Sci. 13, 15-22. doi: 10.4142 /jvs.2012.13.1.15 
Chen, L., Xu, S. Z., Shan, H., and Wang, H. (2011). Investigation on cow cryptosporidiosis in some regions of Yantai. Progress Vet. Med. 32, 114-117.

Chen, Y. G., Li, H. S., Dai, M. X., and Zhang, H. R. (1992). Investigation on Cryptosporidium spp. from animals in Xuzhou area. Chin. J. Zoonoses 8, 39-40.

Cui, Z., Wang, R., Huang, J., Wang, H., Zhao, J., Luo, N., et al. (2014). Cryptosporidiosis caused by Cryptosporidium parvum subtype IIdA15G1 at a dairy farm in Northwestern China. Parasit. Vectors 7:529. doi: 10.1186/s13071014-0529-z

Current, W. L., and Reese, N. C. (1986). A comparison of endogenous development of three isolates of Cryptosporidium in suckling mice. J. Protozool. 1986, 98-108. doi: 10.1111/j.1550-7408.1986.tb05567.x

Esteban, E., and Anderson, B. C. (1995). Cryptosporidium muris: prevalence, persistency, and detrimental effect on milk production in a drylot dairy. J. Dairy Sci. 7, 1068-1072. doi: 10.3168/jds.S0022-0302(95)76723-6

Feng, Y., Ortega, Y., He, G., Das, P., Xu, M., Zhang, X., et al. (2007). Wide geographic distribution of Cryptosporidium bovis and the deer-like genotype in bovines. Vet. Parasitol. 144, 1-9. doi: 10.1016/j.vetpar.2006.10.001

Feng, Y., Wang, L., Duan, L., Gomez-Puerta, L. A., Zhang, L., Zhao, X., et al. (2012). Extended outbreak of cryptosporidiosis in a pediatric hospital, China. Emerg. Infect. Dis. 18, 312-314. doi: 10.3201/eid1802.110666

Guo, S. L., Yuan, F. S., Yu, H., Yu, A. K., Han, Y. M., Wang, C. L., et al. (1993). Investigation on cryptosporidiosis in cattle in Jinan area. Chin. J. Infect. Dis. Livest. poult. 1, 34-35.

Huang, J., Yue, D., Qi, M., Wang, R., Zhao, J., Li, J., et al. (2014). Prevalence and molecular characterization of Cryptosporidium spp. and Giardia duodenalis in dairy cattle in Ningxia, northwestern China. BMC Vet. Res. 10:292. doi: 10.1186/s12917-014-0292-6

Kotloff, K. L., Nataro, J. P., Blackwelder, W. C., Nasrin, D., Farag, T. H., Panchalingam, S., et al. (2013). Burden and aetiology of diarrhoeal disease in infants and young children in developing countries (the Global Enteric Multicenter Study, GEMS): a prospective, case-control study. Lancet 382, 209-222. doi: 10.1016/S0140-6736(13)60844-2

Li, F., Wang, H., Zhang, Z., Li, J., Wang, C., Zhao, J., et al. (2016). Prevalence and molecular characterization of Cryptosporidium spp. and Giardia duodenalis in dairy cattle in Beijing, China. Vet. Parasitol. 219, 61-65. doi: 10.1016/j.vetpar. 2016.01.023

Li, N., Xiao, L., Alderisio, K., Elwin, K., Cebelinski, E., Chalmers, R., et al. (2014). Subtyping Cryptosporidium ubiquitum, a zoonotic pathogen emerging in humans. Emerg. Infect. Dis. 20, 217-224. doi: 10.3201/eid2002.121797

Li, P., Cai, J., Cai, M., Wu, W., Li, C., Lei, M., et al. (2016). Distribution of Cryptosporidium species in Tibetan sheep and yaks in Qinghai, China. Vet. Parasitol. 215, 58-62. doi: 10.1016/j.vetpar.2015.11.009

Liu, A., Wang, R., Li, Y., Zhang, L., Shu, J., Zhang, W., et al. (2009). Prevalence and distribution of Cryptosporidium spp. in dairy cattle in Heilongjiang Province, China. Parasitol. Res. 105, 797-802. doi: 10.1007/s00436-009-1457-2

Ma, J., Cai, J., Ma, J., Feng, Y., and Xiao, L. (2014). Occurrence and molecular characterization of Cryptosporidium spp. in yaks (Bos grunniens) in China. Vet. Parasitol. 202, 113-118. doi: 10.1016/j.vetpar.2014.03.030

Ma, J., Li, P., Zhao, X., Xu, H., Wu, W., Wang, Y., et al. (2015). Occurrence and molecular characterization of Cryptosporidium spp. and Enterocytozoon bieneusi in dairy cattle, beef cattle and water buffaloes in China. Vet. Parasitol. 207, 220-227. doi: 10.1016/j.vetpar.2014.10.011

Mi, R., Wang, X., Li, C., Huang, Y., Zhou, P., Li, Z., et al. (2013). Prevalence and genetic characterization of Cryptosporidium in yaks in Qinghai Province of China. PLOS ONE 8:e74985. doi: 10.1371/journal.pone.0074985

O'Hara, S. P., and Chen, X. M. (2011). The cell biology of Cryptosporidium infection. Microbes Infect. 13, 721-730. doi: 10.1016/j.micinf.2011.03.008

Qi, M., Wang, R., Jing, B., Jian, F., Ning, C., and Zhang, L. (2016). Prevalence and multilocus genotyping of Cryptosporidium andersoni in dairy cattle and $\mathrm{He}$ cattle in Xinjiang, China. Infect. Genet. Evol. 44, 313-317. doi: 10.1016/j.meegid. 2016.07.022

Qi, M. Z., Fang, Y. Q., Wang, X. T., Zhang, L. X., Wang, R. J., Du, S. Z., et al. (2015). Molecular characterization of Cryptosporidium spp. in pre-weaned calves in Shaanxi Province, north-western China. J. Med. Microbiol. 64, 111-116. doi: 10.1099/jmm.0.079327-0

Qin, J. H., Zhao, Y. L., Hao, C. W., Li, C. X., Li, D., Chen, S. H., et al. (1994). Cryptosporidium infections in diarrheal calves in Zhangjiakou city. Chin. J. Vet. Sci. 2, 150.
Qin, S. Y., Zhang, X. X., Zhao, G. H., Zhou, D. H., Yin, M. Y., Zhao, Q., et al. (2014). First report of Cryptosporidium spp. in white yaks in China. Parasit. Vectors 7:230. doi: 10.1186/1756-3305-7-230

Reduker, D. W., Speer, C. A., and Blixt, J. A. (1985). Ultrastructure of Cryptosporidium parvum oocysts and excysting sporozoites as revealed by high resolution scanning electron microscopy. J. Protozool. 32, 708-711. doi: 10.1111/j.1550-7408.1985.tb03106.x

Robertson, L. J., Björkman, C., Axén, C., and Fayer, R. (2014). “Cryptosporidiosis in farmed animals," in Cryptosporidium: Parasite and Disease, eds S. M. Cacciò and G. Widmer (Berlin: Springer), 149-236.

Ryan, U., Fayer, R., and Xiao, L. (2014). Cryptosporidium species in humans and animals: current understanding and research needs. Parasitology 141, 1667-1685. doi: 10.1017/S0031182014001085

Santín, M., Trout, J. M., and Fayer, R. (2008). A longitudinal study of cryptosporidiosis in dairy cattle from birth to 2 years of age. Vet. Parasitol. 155 , 15-23. doi: 10.1016/j.vetpar.2008.04.018

Su, Y., Bai, G. Y., Sun, X. D., Liu, Y., Wang, C. R., Zhang, J., et al. (2011). Characterization of Cryptosporidium spp. from preweaned calves of Jilin and Daqing area by $18 \mathrm{~S}$ rRNA gene nested PCR-RFLP. Chin. J. Vet. Sci. 31, 347-351.

Trout, J. M., and Santín, M. (2008). "Livestock," in Cryptosporidium and Cryptosporidiosis, eds R. Fayer and L. Xiao (Boca Raton, FL: CRC Press), 451-483.

Vinayak, S., Pawlowic, M. C., Sateriale, A., Brooks, C. F., Studstill, C. J., BarPeled, Y., et al. (2015). Genetic modification of the diarrhoeal pathogen Cryptosporidium parvum. Nature 523, 477-480. doi: 10.1038/nature14651

Wang, L., Zhang, H., Zhao, X., Zhang, L., Zhang, G., Guo, M., et al. (2013). Zoonotic Cryptosporidium species and Enterocytozoon bieneusi genotypes in HIV-positive patients on antiretroviral therapy. J. Clin. Microbiol. 51, 557-563. doi: 10.1128/JCM.02758-12

Wang, R., Jian, F., Zhang, L., Ning, C., Liu, A., Zhao, J., et al. (2012). Multilocus sequence subtyping and genetic structure of Cryptosporidium muris and Cryptosporidium andersoni. PLOS ONE 7:e43782. doi: 10.1371/journal.pone. 0043782

Wang, R., Li, G., Cui, B., Huang, J., Cui, Z., Zhang, S., et al. (2014a). Prevalence, molecular characterization and zoonotic potential of Cryptosporidium spp. in goats in Henan and Chongqing, China. Exp. Parasitol. 142, 11-16. doi: 10.1016/ j.exppara.2014.04.001

Wang, R., Ma, G., Zhao, J., Lu, Q., Wang, H., Zhang, L., et al. (2011a). Cryptosporidium andersoni is the predominant species in post-weaned and adult dairy cattle in China. Parasitol. Int. 60, 1-4. doi: 10.1016/j.parint.2010. 09.002

Wang, R., Qiu, S., Jian, F., Zhang, S., Shen, Y., Zhang, L., et al. (2010). Prevalence and molecular identification of Cryptosporidium spp. in pigs in Henan, China. Parasitol. Res. 107, 1489-1494. doi: 10.1007/s00436-010-2024-6

Wang, R., Wang, H., Sun, Y., Zhang, L., Jian, F., Qi, M., et al. (2011b). Characteristics of Cryptosporidium transmission in preweaned dairy cattle in henan, China. J. Clin. Microbiol. 49, 1077-1082. doi: 10.1128/JCM.02194-10

Wang, R., Zhang, L., Axén, C., Bjorkman, C., Jian, F., Amer, S., et al. (2014b). Cryptosporidium parvum IId family: clonal population and dispersal from Western Asia to other geographical regions. Sci. Rep. 4:4208. doi: 10.1038/ srep04208

Wang, R., Zhang, L., Feng, Y., Ning, C., Jian, F., Xiao, L., et al. (2008). Molecular characterization of a new genotype of Cryptosporidium from American minks (Mustela vison) in China. Vet. Parasitol. 154, 162-166. doi: 10.1016/j.vetpar. 2007.12.038

Wang, R., Zhang, X., Zhu, H., Zhang, L., Feng, Y., Jian, F., et al. (2011c). Genetic characterizations of Cryptosporidium spp. and Giardia duodenalis in humans in Henan, China. Exp. Parasitol. 127, 42-45. doi: 10.1016/j.exppara.2010. 06.034

Watanabe, Y., Yang, C. H., and Ooi, H. K. (2005). Cryptosporidium infection in livestock and first identification of Cryptosporidium parvum genotype in cattle feces in Taiwan. Parasitol. Res. 97, 238-241. doi: 10.1007/s00436-0051428-1

Xiao, L. (2010). Molecular epidemiology of cryptosporidiosis: an update. Exp. Parasitol. 124, 80-89. doi: 10.1016/j.exppara.2009.03.018

Xiao, L. H., Fayer, R., Ryan, U., and Upton, S. J. (2004). Cryptosporidium Taxonomy: recent advances and implications for public health. Clin. Microbiol. 17, 72-97. doi: 10.1128/CMR.17.1.72-97.2004 
Xiao, L. H., and Ryan, U. (2008). "Molecular epidemiology," in Cryptosporidium and Cryptosporidiosis, eds R. Fayer and L. Xiao (Boca Raton, FL: CRC Press), 119-172.

Zahedi, A., Phasey, J., Boland, T., and Ryan, U. (2016). First report of Cryptosporidium species in farmed and wild buffalo from the Northern Territory, Australia. Parasitol. Res. 115, 1349-1353. doi: 10.1007/s00436-0164901-0

Zhang, W., Wang, R., Yang, F., Zhang, L., Cao, J., Zhang, X., et al. (2013). Distribution and genetic characterizations of Cryptosporidium spp. in preweaned dairy calves in Northeastern China's Heilongjiang Province. PLOS ONE 8:e54857. doi: 10.1371/journal.pone.0054857

Zhang, X. X., Tan, Q. D., Zhou, D. H., Ni, X. T., Liu, G. X., Yang, Y. C., et al. (2015). Prevalence and molecular characterization of Cryptosporidium spp. in dairy cattle, northwest China. Parasitol. Res. 114, 2781-2787. doi: 10.1007/s00436015-4537-5

Zhao, G. H., Ren, W. X., Gao, M., Bian, Q. Q., Hu, B., Cong, M. M., et al. (2013). Genotyping Cryptosporidium andersoni in cattle in Shaanxi province, northwestern China. PLOS ONE 8:e60112. doi: 10.1371/journal.pone.0060112
Zhou, R., Li, G., Xiao, S., Xia, Y., and Guo, Y. (2007). PCR amplification and sequence analyses of ITS-1 rDNA from Cryptosporidium andersoni in dairy cattle. Parasitol. Res. 100, 1135-1138. doi: 10.1007/s00436-0060358-x

Zhou, S. W., Zhang, Y., Ji, D. C., and Qi, T. Y. (1985). Investigation on cryptosporidiosis in calves and cross-transmission study. Chin. J. Prev. Vet. Med. 3, 21-22.

Conflict of Interest Statement: The authors declare that the research was conducted in the absence of any commercial or financial relationships that could be construed as a potential conflict of interest.

Copyright (c) 2017 Wang, Zhao, Gong and Zhang. This is an open-access article distributed under the terms of the Creative Commons Attribution License (CC BY). The use, distribution or reproduction in other forums is permitted, provided the original author(s) or licensor are credited and that the original publication in this journal is cited, in accordance with accepted academic practice. No use, distribution or reproduction is permitted which does not comply with these terms. 